\title{
PESSOAS ACAMADAS SOB O CUIDADO DAS EQUIPES DE SAÚDE DA FAMIILIA NO DISTRITO SANITÁRIO NORTE DE CAMPO GRANDE/MS: PREVALÉNCIA, AVALIAÇÃO DO ACESSO AOS SERVIÇOS DE SAÚDE, INDEPENDÉNCIA FUNCIONAL E SOBRECARGA DOS CUIDADORES
}

\author{
Arthur de Almeida Medeiros \\ Gustavo Christofoletti \\ Orientador \\ Mara Lisiane de Moraes dos Santos \\ Coorientadora
}

\author{
Programa de Pós-Graduação em Saúde e Desenvolvimento da Região Centro-Oeste \\ Universidade Federal de Mato Grosso do Sul
}

$\mathrm{A}^{\mathrm{s}}$ s modificações dos padrões de morbidade, mortalidade e invalidez têm gerado impactos importantes no cenário atual da saúde e despertado debates indispensáveis para o cuidado integral de toda a população. Esta tese teve por objetivo estudar os usuários acamados assistidos pela Estratégia Saúde da Família (ESF) e seus principais cuidadores. Trata-se de um estudo transversal de base populacional no qual foram convidados a participar os usuários acamados e seus cuidadores residentes nas regiões urbanas com cobertura da ESF no distrito sanitário norte de Campo Grande/MS. Procedeu-se com entrevistas domiciliares nas quais foi aplicado um instrumento com variáveis clínicas, socioeconômicas e demográficas relacionadas com os usuários acamados e seus cuidadores, além da versão brasileira da escala Burden Interview, para avaliação da sobrecarga dos cuidadores, e da Medida de Independência Funcional (MIF). Para a análise estatística, cujas variáveis atenderam aos critérios de normalidade, foram utilizados os testes de qui-quadrado e ANOVA com pós-teste de Tukey. As variáveis que não apresentaram padrão de normalidade foram analisadas utilizando-se a correlação de Spearman, o teste $\mathrm{U}$ de Mann-Whitney e o teste de Kruskal-Wallis com pós-teste de Tukey. Em todas as análises, foi admitido um nível de significância de $5 \%$ para distinção da hipótese alternativa em relação à hipótese nula. Os resultados mostram que em relação aos usuários acamados $(n=283)$, a maioria era de mulheres $(n=169 / 59,7 \%)$, com disfunção neurológica ( $n=149 / 52,7 \%)$, nível so- cioeconômico classe C ( $n=164 / 58,0 \%)$, que não foram encaminhadas a nenhum serviço de reabilitação (n145/51,2\%) e que apresentaram mediana da MIF total de 79 pontos, classificados com dependência modificada - com necessidade de auxílio de até $25 \%$ para a realização de suas atividades. Entre os usuários que foram encaminhados a algum serviço de reabilitação, a fisioterapia foi o que mais recebeu demanda por encaminhamento ( $n=124 / 89,8 \%)$. Verificou-se, ainda, que a falta de vagas e a dificuldade de locomoção/transporte foram os principais limitadores tanto para o acesso aos serviços de reabilitação quanto para a continuidade dos atendimentos. Quanto aos cuidadores $(n=218)$, identificou-se que a mediana de idade foi de 53 anos e que a maioria foi do sexo feminino $(n=190 / 87,2 \%)$, sendo mais frequentes as filhas $(n=71 / 32,6 \%)$, seguidas das mães $(n=46 / 21,1 \%)$ e das esposas $(n=25 / 11,5 \%)$. Foi observado que os cuidadores apresentavam nível de sobrecarga moderada e que teve associação negativa significativa entre a sobrecarga dos cuidadores e a Medida de Independência Funcional. Os dados apontam para urgente necessidade de (re)estruturação da rede de atenção destinada aos usuários acamados e de reorganização do processo de trabalho, bem como da necessidade de ampliação do olhar para o reconhecimento das necessidades de saúde dos usuários acamados e de seus cuidadores.

Palavras-chave: Acesso aos serviços de saúde. Cuidadores. Incapacidade funcional. Pacientes domiciliares. Atenção primária à saúde. 